\title{
OBSERVATION OF IONIZATION OF LASER EXCITED ATOMS BY SYNCHROTRON RADIATION
}

J.M. Bizau, F. Wuilleumier, P. Gerard, P. Dhez Laboratoire de Spectroscopie Atonique et Ionique and Laboratoire pour l'Utilization du Rayonnemet Electronagnetique Universite Paris-Sud, Orsay, France 91405

B. Carre, G. Spiess

Service de Physique des Atomes et des Surfaces CEN, Saclay, France 91191

\section{D.L. Ederer}

Radiation Physics Division

National Bureau of Standards

Gaithersburg, M 20899

\author{
J.L. Picqué, J.L. LeGouet, J.C. Keller \\ Laboratoire Aime Cotton \\ CNRS, Orsay, France 91405 \\ P. Koch \\ Physics Dept. \\ State University of New York \\ Stony Brook, NY 11790
}

We have begun a program to measure oscillator strengths of autoionizing resonances that result froin a transition in the VUV between a 1 aser excited initial state and a final state in which a core electron is promoted. These measurements demonstrate a new technique to combine synchrotron radiation, laser pumping, and photcelectron spectroscopy.

Measurements of the energy positions of autoionizing resonances have been honed to a fine art over the past 50 years. ${ }^{1}$ Total cross section measurements and the parameters that describe autoionizing resonances have been determined. ${ }^{2}$ Most of these studies have been made from the dipole allowed ground state. Recently autoionizing resonances have been observed from excited initial states ${ }^{3}$ and from ion initial states. ${ }^{4}$ We have heard several talk $s^{5},{ }^{6}$ at this meeting which described some of this type of research. In the measurenents to be described in this paper, laser radiation is combined with synchrotron radiation, as shown schematicaly in Figure 1, to study the photoionization from excited initial states to continuum final states or to autoionizing final states. Continuum radiation from the Aneau de Collisions d'Orsay (ACO), which is installed at the Universite de Paris-Sud,in Orsay France, is inonochromatized by a toroidal grating monochromator (TGM) 7 and is focused by a toroidal output mirror on to a weakly collimated sodium beam emanating from a furnace mounted on the axis of a cylinderical mirror analyzer (CMA). This electron spectroineter is used to study the kinetic energy distribution of the ejected photoelectrons produced by the interaction of the photon beam with the focused synchrotron radiation. 
This arrangement has been used to study atoms ${ }^{8}$ and molecules ${ }^{9}$ in the ground state.

To extend this research to excited states ${ }^{0}$, we have mounted a ring dye laser (maximum intensity at the sodium $D$ lines $9 \mathrm{w} / \mathrm{cm}^{2}$ ) so that its beam traverses the CMA in a direction perpendicular to the CMA axis at the focus of the synchrotron radiation. The laser is stabilized to $20 \mathrm{MHz}$ by locking it to the $3^{2} S_{1 / 2}(F=2)+32 P_{3 / 2}(F=3)$ transition in an auxiliary collimated sodium beam also shown schematically Figure 1 . The size of the laser bean is adjusted so that it just fills the source volume, $V$. Electrons emanating from this volume at the magic angle $\left(54^{\circ} 48^{\prime}\right)$ can be detected.

We have chosen to determine the oscillator strength of a group of autoionizing resonances in sodium il with the same parity as the ground state.

The area $N(h v)$ of the photoelectron peak from $2 p$ ionization of the 1aserexcited atoms is proporational to the product of the excited state density and the photoionization cross section $\sigma(h v)^{12}$ :

$$
N(h v)=K \cdot I(h v) \cdot E(h v) \cdot n(3 p) \cdot \int_{\Delta E} \sigma\left(h v^{\prime}\right) \cdot W\left(h v-h v^{\prime}\right) d\left(h v^{\prime}\right)
$$

where $K$ is the spectrometer constant, $I(t w)$ is the photon flux at photon energy hu; $E(h v)$ is th kinetic energy of the photoelectron; $n(3 p)$ is the density of laser-excited atoms; $\Delta E$ is the monochromator band pass, and $W$ is the photon monochromator window function. Similarly, the integrated area $N\left(h v_{R}\right)$, of the photoelectron peak for the autoionizing resonance is given by:

$$
N\left(h \nu_{R}\right)=K_{R} \cdot I\left(h \nu_{R}\right) \cdot E \cdot\left(h \nu_{R}\right) \cdot n(3 p) \cdot f_{R}^{\prime}
$$

where $I\left(h v_{R}\right)$ is the photon flux at the resonance excitation energy, $E\left(h \nu_{R}\right)$ is the kinetic energy of the decay electron and $\Delta E_{R}$ is the monochromator bandpass. In our experiment, the monochromator bandpass (about $0.1 \mathrm{eV}$ in first order) was much larger than the width of the resonance, and the integral over the oscillator strength density in Eq(2) can be replaced by the oscillator strength of the resonance. The oscillator strength can be obtained from the ratio of Eq(2) to Eq(1), yielding:

$$
f_{R}=\frac{N\left(h v_{R}\right)}{N(h v)} \cdot \frac{K}{K_{R}} \cdot \frac{I(h v)}{I\left(h v_{R}\right)} \cdot \frac{E(h v)}{E\left(h_{R}\right)} \cdot \Delta E \cdot \sigma(h v)
$$

This ratio is independent of the excited state density, one of the more difficult quantities to determine experimentally. All the terms on the right of $E q(3)$ can be determined experimentally, but one can obtain a 
very good approximate value of $f_{B}$ by assuming $\sigma\left(h_{\nu}\right)$, the excited state continuum cross section, is equal to the photoionization for the ground state. 13

In Figure 3 we plot $\mathrm{Eq}(3)$ as a function of photon energy for some autoionizing resonances in sodium vapor. Photoionization cross sections froin an excited ground state in 1 ithium and barium have also been obtained.

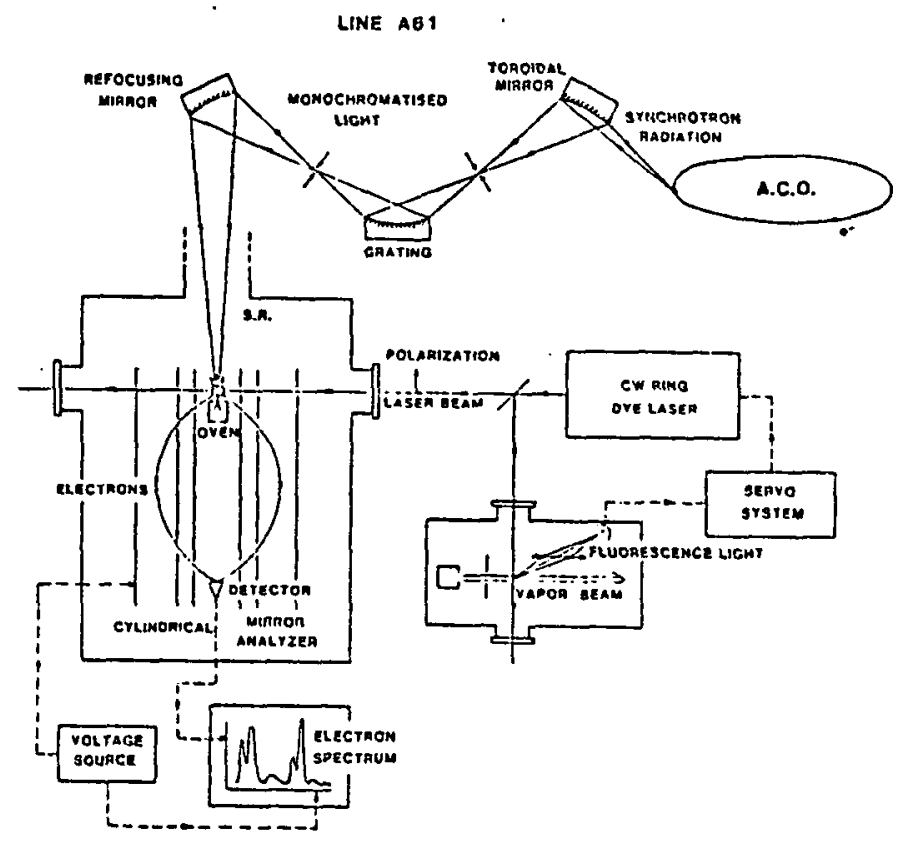

F19. 1

A schematic representation of the apparatus used for synchrotron radiation studies of laser-eaxcited states. XUY radiation from the ACO storage ring at the Laboratoire pour 1-Utilization du Rayonnement El ectro-magnetique (LURE) is monochromatized and. Is focused on the axis of a cylindrical mirror electron energy analyzer (CMA). An oven located on the CMA axis

produces an effusive cloud of sodium which is excited by the stabtlized laser bean trayersing the CMA perpendicular to its axis. the beam

from the ring dye laser $\left(9 \mathrm{kcm}^{2}\right.$ max power) is stabilized to $20 \mathrm{MHz}$ by using fluorescence from the $3^{2} S_{1 / 2}(F=2)$ ${ }^{2} P_{3 / 2}(F=3)$ transition in a well

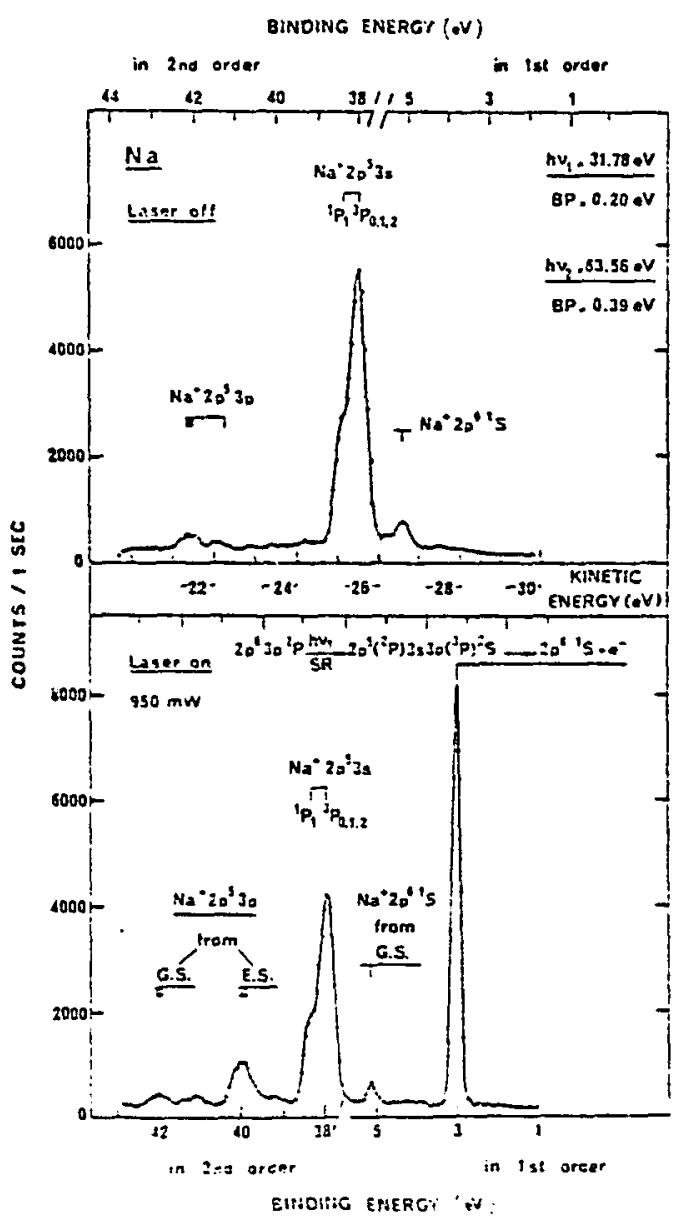

Fig. 2

Photolectron energy spectrum taken with, the TGI set at $31.78 \mathrm{eV}$. The intense peak at about $29 \mathrm{eV}$ kinetic energy (KE) corresponds to the autotonizing decay of the resonance excited by the inner subshell transition indicated in the figure. The small peak at 26.5 ey $\mathrm{KE}$ is due to the direct ionization of the 3s electron by the $31.78 \mathrm{eV}$ photons. The structures at $25.5 \mathrm{ev}, 22.1 \mathrm{eV}$, - and at $21.4 \mathrm{eV} K E$ are due to the direct ionization of the $2 p^{5} 3$ s subshell. by 63.56 ev radiation (2nd order radiation diffracted by the $T(M)$. The struc ture in the lower frame at $23 \mathrm{eV}$ $X E$ is due to the ionization of a $2 p$ electron in the laser excited sodium 
Fig. 3

Excitation function of laser excited sodium as a function of photon energy for a TGM Dand pass of $0.10 \mathrm{eV}$ (BP in figure). The configuration and terms of the autoionizing resonance in the spectral range shown in the figure are indicated according to Ref. 11. The energy position of the $2 p^{5}(2 p) 3 s 3 p(1 p)^{2} D_{3 / 2}$ differs by 0.022 ey from that of Ref. 11. The width of the structure $r=0.11$ includes the resonance widths plus the TGY bandpass.

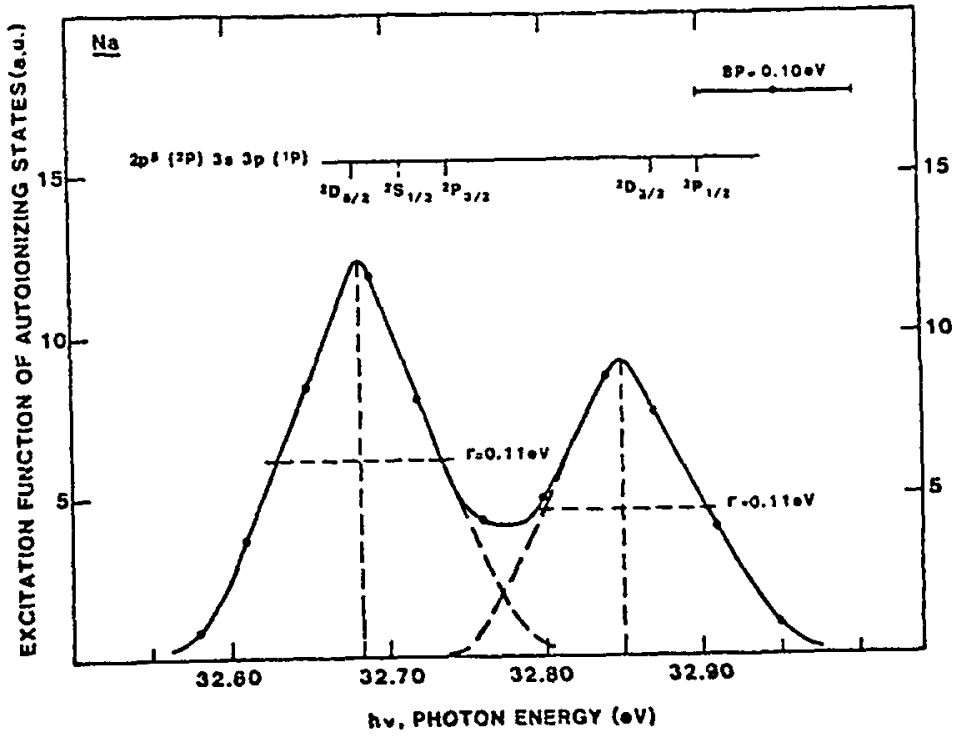

\section{References}

1. H. Beutler, Z. Physics 93, 177 (1935); K. Codling, Rep. Prog. Physics 36, 541 (1973).

2. M.0. Krause, F. Cerrina, and T. Fahiman, Phys. Rev. Lett. 50, 118 (1983); J.A.R. Samson, Phys. Lett. 28C 303 (1976).

3. W. Hill, III, K.T. Cheng, H. Johnson, T.B. Lucatorto, T.J. McIlrath, and J. Sugar, Physical Rev. Lett. 49,1634 (1982).

4. T.J. Mcll rath and T.8. Lucatorto, Phys. Rev. Lett. 38, 1390 (1977).

5. M.A. Baig, J.P. Connerade, H.R.S. Garton. J. Hornes, C. Maynew, G. Noldeke, and $K$. Sommer, these proceedings.

6. T.J. MeIlrath, C. Cromer, V. Kaufman, J. Sugar, H.T. Hill, III, and D. Cooper, these proceedings.

7. P.X. Larsen, H.A.M. van Bers, J.M. Bizau, F. Huilleumier, V. Schmidt, and D.L. Ederer, Nucl. Instrum. Meth. 195,245 (1982).

8. S. Krummacher, Y. Schmidt, J.M. 3izau, O.L. Ederer, P. Ohez, and F. Wuilleumier, J. Phys. B. 15, 4363 (1982).

9. S. Krumacher, V. Schmidt, F. Wuilleumier, J.M. Bizau, and 0.L. Ederer, j. Phys. B. 16,1733 (1983).

10. J.L. Legou'et, J.L. Picqué, F. Wuilleunier, J.M. Bizau, P. Dhez, P. Koch, and D.L. Ederer. Phys. Rev. Lett. 48,600 (1982).

11. J. Sugar, T.B. Lucatorto, T.J. McIlrath, A.H. Weiss, Opt. Lett. 4,109 (1979).

12. M.0. Krause, Chem. Phys. Lett. 10, 65 (1971).

13. T.N. Chang and Y.S. Kim, J. Phys. B. 15, L835 (1982). 